\title{
The performance of iPad-based noise-field perimeter versus Humphrey Field Analyser in detecting glaucomatous visual field loss
}

\author{
Jianbin Ding $\mathbb{1}^{1,2} \cdot$ Ivan C. Tecson ${ }^{2,3} \cdot$ Bryan C. H. Ang ${ }^{2} \cdot$ Wenqi Chiew $\mathbb{B}^{4} \cdot$ Chunhau Chua $^{2} \cdot$ Leonard W. L. Yip $\mathbb{D}^{1,2}$
}

Received: 20 May 2020 / Revised: 28 February 2021 / Accepted: 6 April 2021 / Published online: 20 April 2021

(c) The Author(s), under exclusive licence to The Royal College of Ophthalmologists 2021

\begin{abstract}
Background To conduct a preliminary evaluation of the feasibility of visual field fast (VFF), a free iPad-based noise-field perimeter, in detecting glaucomatous scotomas versus the clinical-standard Humphrey visual field (HVF) test. VFF confronts subjects with a screen of flickering stimulus, allowing the immediate perception of scotomas.

Methods This was a cross-sectional observational study of 66 glaucoma patients (66 eyes) and 30 healthy controls (30 eyes). All patients had no other visual field disorders. VFF was compared against HVF in terms of whole field and quadrants for the following: (1) correspondence in scotoma detection. (2) Agreement and correlation of the scotoma size (percentage of abnormal visual field area). (3) Test duration. Other domains tested included: (1) correlation of VFF scotoma area with the severity of visual field loss on HVF (mean deviation, MD; visual field index, VFI). (2) Repeatability of VFF. (3) Patient descriptors of scotomas.

Results Using HVF pattern deviation plot as a reference, VFF detected 52/57 (91.2\%) of glaucoma subjects with 1 falsepositive (control) $(\mathrm{kappa}=0.86) .146 / 184(79.3 \%)$ of abnormal quadrants (visual field defect present) were localized and 23/ $157(14.6 \%)$ healthy quadrants were falsely identified as abnormal (kappa $=0.61)$. VFF underestimated scotoma area as compared to HVF $(21.0 \%$ versus $44.0 \%, p<0.01)$ but correlated positively $(r=0.268, p=0.044)$ with HVF area and negatively with VFI $(r=-0.340, p=0.01)$ and MD $(r=-0.398, p<0.01)$. Using HVF total deviation plot as reference, VFF's glaucoma detection rate remained unchanged (kappa $=0.86$ ) with similar quadrant detection (kappa $=0.68$ ). However, a greater underestimation of scotoma area was observed $(21.0 \%$ versus $85.4 \%, p<0.01)$. VFF's quantitative repeatability was excellent for whole field (intraclass correlation coefficient, ICC: $0.96 ; p<0.0001$ ) and quadrants (ICC: 0.82-0.96; all $p<0.001)$. Qualitatively, 35/37 (94.6\%) of subjects reported reduced luminance and flicker in scotomas, with similar morphologies on retests. VFF is faster than HVF SITA-Standard in glaucoma $(3.60 \pm 1.85$ min versus $6.92 \pm$ $1.12 \mathrm{~min}, p<0.01)$ and control $(1.12 \pm 0.486 \mathrm{~min}$ versus $5.16 \pm 0.727 \mathrm{~min}, p<0.01)$.

Conclusion This early model of VFF accurately detected scotomas with high repeatability. However, its accuracy in localizing and quantifying the scotoma can be improved. Considering its portability and cost-effectiveness, VFF demonstrated potential as a general screening tool for moderate-to-severe glaucoma.
\end{abstract}

Supplementary information The online version contains supplementary material available at https://doi.org/10.1038/s41433021-01541-x.

Leonard W. L. Yip

leonard_yip@ttsh.com.sg

1 Lee Kong Chian School of Medicine, Nanyang Technological University, Singapore, Singapore

2 Department of Ophthalmology, Tan Tock Seng Hospital, Singapore, Singapore

3 Department of Ophthalmology, Cardinal Santos Medical Centre, Manila, Philippines

4 Yong Loo Lin School of Medicine, National University of Singapore, Singapore, Singapore

\section{Introduction}

Glaucoma is the third leading cause of global blindness, behind cataract and uncorrected refractive error [1]. In 2013, glaucoma's global prevalence was 64.3 million (3.54\%) in the 40-80 years age group [2]. This figure was estimated to have hit 76.0 million by 2020, posing a significant economic burden proportional to disease severity [1,3]. Although early intervention can slow or arrest this irreversible deterioration, the disease is often insidious and remains unnoticed until later stages. In Singapore, at least $66 \%$ of glaucoma patients are believed to be undiagnosed [4]. In developing countries like India, this figure reaches an alarming 94\% [5]. 
In Singapore, more than half of the undiagnosed glaucoma cases suffer at least moderate visual field (VF) loss [defined as a Humphrey visual field (HVF) mean deviation (MD) of $>-6.0 \mathrm{~dB}$ ] [4]. These patients will have apparent perimetric findings. However, the current clinical standard of assessment using the HVF Analyser is only available in ophthalmic units and not designed for population screening.

Unfortunately, population-based screening for glaucoma remains a challenge, both technically and practically. A previous meta-analysis on potential screening tools has failed to identify a superior modality [6]. Ocular imaging and perimetry require specialized machines, while tonometry and ophthalmoscopy, although cheaper, can only be conducted by trained examiners. Furthermore, the latter two tests lack sensitivity unless used in combination $[7,8]$.

Noise-field campimetry, or entopic (snowfield) perimetry, is a method to simultaneously test the entire VF by confronting patients with a screen of randomly flickering, black and white stimulus [9]. This is faster than the pointto-point testing used in standard automated perimetry and has been proven effective in detecting scotomas due to cytomegalovirus retinitis, diabetic retinopathy, and glaucoma [10-12]. However, the practicality aspect was compromised by the bulky testing setup [9-12]. Our group has developed a noise-field testing iPad application (app), named 'visual field fast' (VFF) (Leonard Yip, Apple App Store), enhancing the test's portability. VFF is available for free on the Apple App Store. VFF operates on any iPad running iOS 10.0 (Sep 2016) or higher, covering the same VF area as the 24-2 HVF assessment. Patients can immediately perceive scotomas as zones of abnormal flickering in terms of colour and frequency [9], and delineate them using the touch screen for automated quantification of the percentage of VF loss. The frequency (not exceeding the iPad's screen refresh rate of $120 \mathrm{~Hz}$ ), size, and colour of stimulus can be adjusted. Due to the variety of stimulus available in this novel perimeter, this preliminary study aims to empirically derive a recommended test stimulus for VFF and subsequently evaluate its feasibility in detecting glaucomatous VF loss.

\section{Methods}

This cross-sectional observational study was conducted at a tertiary referral eye centre in Singapore, from May to September 2019. This study adhered to the tenets of the Declaration of Helsinki, and ethics approval was obtained from the Domain-Specific Review Board. Signed informed consent was obtained from all subjects.

\section{Study population}

All subjects were older than 21 years (median: 68.5; range: 39-90), with SITA-Standard 24-2 HVF test (HVF Analyser III, Carl Zeiss Meditec, Dublin, CA, USA) performed within 1 month before the day of recruitment.

The study group comprised of subjects with an established diagnosis of glaucoma on follow up. Exclusion criteria included pre-perimetric disease, subjects with 10-2 HVF test, non-glaucomatous causes of VF loss, macular diseases, and unreliable HVF results. The control group comprised of normal subjects with no known VF defects. Inclusion criteria included reliable 24-2 HVF results (glaucoma hemifield test of borderline or better, pattern standard deviation (SD) not $<5 \%$ ) and a best-corrected visual acuity of $6 / 12$ or better on the Snellen's chart. To avoid bias, only one eye per subject was tested at random.

\section{Study phases}

The following were the three adjustable properties of VFF stimulus (Fig. 1A).

(1) Size (pixels/px): $2 \times 2$ (smallest), $4 \times 4,8 \times 8,16 \times 16$, $32 \times 32$ (largest).

(2) Frequency (Hz): 1-30 adjustable scale.

(3) Colour: black-white (BW), red-green, blue-yellow.

The default stimulus setting is $2 \times 2 \mathrm{px}, 30 \mathrm{~Hz}, \mathrm{BW}$.

As VFF supports a range of stimulus, this study was conducted in three phases, with one stimulus property adjusted and tested in each phase. This enables us to empirically derive a suitable stimulus setting for detecting visual field loss. The setting for each phase was:

Phase 1 (determine a favourable stimulus size): variable size, $30 \mathrm{~Hz}, \mathrm{BW}$.

Phase 2 (determine a favourable frequency): recommended size, variable frequency, BW.

Phase 3 (testing of the recommended stimulus): recommended size, recommended frequency, BW.

\section{VFF testing procedures}

Room brightness and screen luminance were measured using a photometer (AS 803 Lux Meter, Smart Sensor, Dongguan, Guangdong, China). VFF testing was performed in a dark room with all light sources switched off ( $<0.1$ lux). The test was performed by two investigators (JD and WC), both masked to the HVF results of glaucoma subjects. A 9.7-inch iPad Pro (Apple, Cupertino, CA, USA) was set to auto-brightness $\left(5 \mathrm{~cd} / \mathrm{m}^{2}\right.$ in the dark room), mounted to a mobile stand (Fig. 1B). 

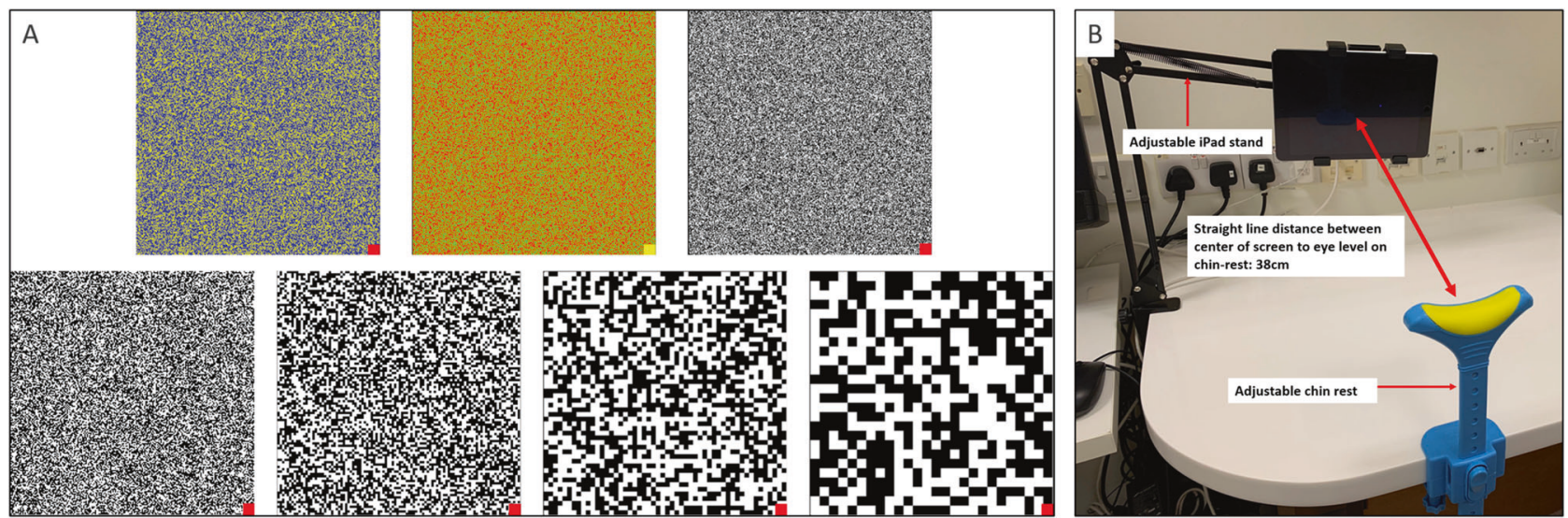

Fig. 1 VFF testing stimulus and experimental setup. A Stimulus used for VFF testing. Top column (left to right): blue-yellow, red-green and black-white stimulus of $2 \times 2$ pixel size. Bottom column (left to right): variable stimulus size $4 \times 4,8 \times 18,16 \times 16,32 \times 32$. B Display of experimental setup used for VFF testing.
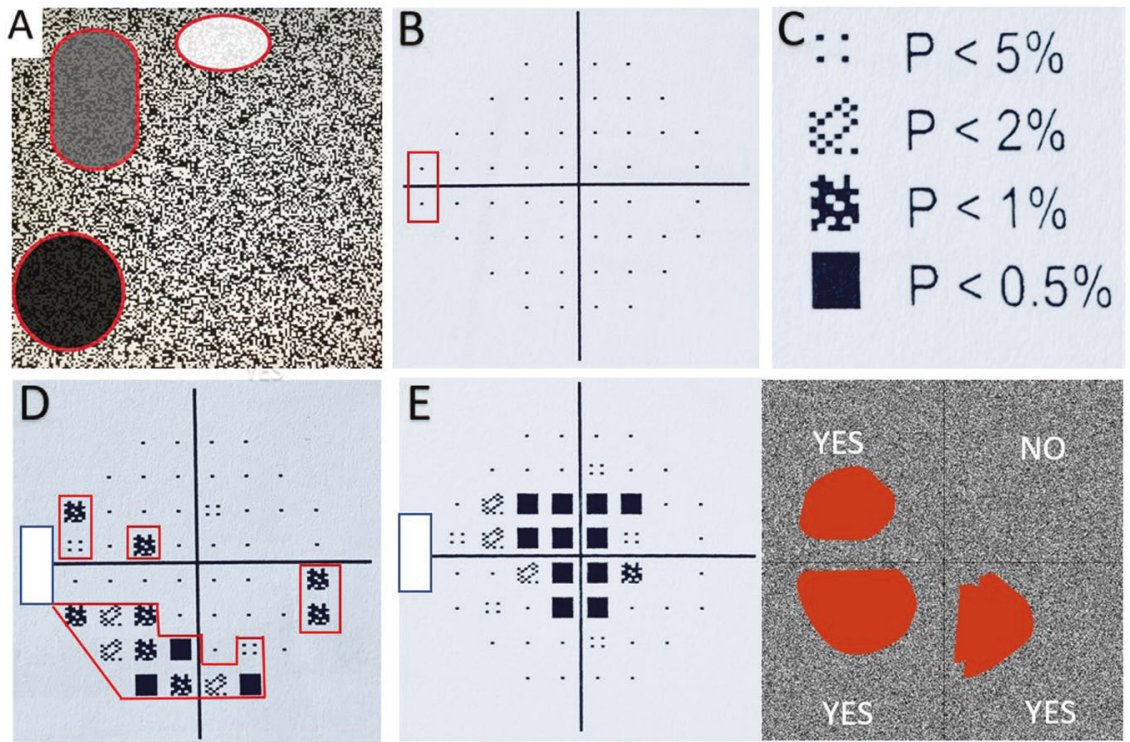

Fig. 2 Compilation of HVF pattern deviation plots, grey scale, and HVF results to illustrate steps of data interpretation. A Pictorial illustration of patient perception of scotoma on VFF. The perceived scotomas may vary in brightness, as depicted by the three circled regions. These areas of abnormality will be perceived instantaneously due to the stark contrast with the noise-field background. B Red box indicated the two nasal-most HVF spots to be excluded for perfect

As visual field loss in glaucoma commonly starts from the peripheries, corresponding to the boundaries of the iPad screen, patients with milder diseases may not realise their scotomas unless specifically instructed to take note. As such, a standardized set of instruction was given to remind patients to consciously pay attention to the corners of the screen and look out for any abnormalities: "while looking at the red dot, look out for areas that are not flashing, flashing lesser, dark, bright, or simply abnormal compared to the rest (Fig. 2A). Take note of the iPad's boundaries and corners. It is okay if everything flashes overlap with VFF testing area. In all subsequent figures, white boxes will be used to block off these two points. C Different appearances of abnormal spots on pattern deviation probability plots. D Illustration of quadrant grading of a subject's right eye. Red boxes indicate scotomas of diseased quadrants. Superotemporal quadrant is graded normal. E Illustration of quadrant correspondence between HVF and VFF. 'No' indicates failure of VFF to detect a diseased quadrant.

normally or equally. Draw any abnormal areas using your fingers'.

The patient rested on a chin-rest with the non-testing eye patched. The iPad was viewed at a distance of $38 \mathrm{~cm}$, which is the farthest working distance capable of accommodating $\pm 24^{\circ}$ of the VF when using a variable fixation target. Given the limited spatial extent of the display, the four quadrants of the VF (superotemporal, inferotemporal, superonasal and inferonasal; ST, IT, SN, IN) were tested by asking observers to successively fixate at each of the four corners of the screen (Supplementary Fig. 1). For patient comfort, the red 
fixation target's position was always kept central at eye level by physically moving iPad between each condition.

After outlining the scotomas, the percentage of VF loss was automatically calculated at two levels: whole field and individual quadrants. Patient descriptors of scotomas were also documented.

\section{HVF interpretation}

Pattern deviation and total deviation probability plots were used for analysis. We analysed the plot at two levels, the entire field and individual quadrants. Points with $p<5 \%$ or less were considered abnormal, as the deviation from normal found at that point location occurs in fewer than $5 \%$ or less of normal subjects (Fig. 2B). The two nasal-most points were excluded to obtain the true overlap between the fields tested by VFF and HVF (Fig. 2C). When the MD threshold was exceeded, the total deviation plot was used.

The entire field of a glaucoma patient was deemed to have VF loss (diseased field) if either of the following criteria was fulfilled [13]:

(1) HVF having $\geq 5$ abnormal points with $\geq 2$ of $p<1 \%$, or

(2) A cluster of $\geq 3$ contiguous abnormal points.

The individual quadrants of a glaucoma patient with an abnormal field were deemed to have VF loss (diseased quadrant) if either of the following criteria was fulfilled (Fig. 2D):

(1) $\geq 2$ points of $P<1 \%$, or

(2) A cluster of $\geq 3$ contiguous points with $P<5 \%$, not crossing the horizontal meridian. Both quadrants (entire hemifield) were considered diseased if the cluster crossed the vertical meridian.

\section{Analysis of scotoma size}

The percentage of abnormal points on the pattern/total deviation plot was taken as a surrogate marker to quantify the extent of VF loss (scotoma area) on HVF. This was done at two levels, the whole field and individual quadrants. Comparison of the scotoma area was made between HVF and VFF.

\section{HVF-VFF scotoma detection correspondence}

Using the HVF pattern/total deviation plot as a reference, we checked for the presence (yes) and absence (no) of corresponding scotoma VFF (Fig. 2E). This was performed for the whole field (detection of 'diseased' fields) and individual quadrants (localization of 'diseased' quadrants) (Fig. 3A).

\section{VFF repeatability assessment}

Test-retest reliability of VFF was assessed at Phase 3, using the empirically derived suitable setting. Comparisons were made quantitatively (scotoma area) and qualitatively (scotoma location).

\section{Statistical methods}

Categorical data were expressed as absolute numbers. Continuous variables were expressed as median with interquartile range for non-parametric and mean $\pm \mathrm{SD}$ for parametric data. Statistical analysis was performed using IBM SPSS Statistics version 22 (IBM Corp, New York, USA). All tests were twotailed, with statistical significance established at $p<0.05$.

Quantitative agreement (scotoma area) between HVF (pattern deviation and total deviation) and VFF was assessed using the Bland-Altman Plot. Differences were further analysed using the Wilcoxon Signed-rank Test.

Qualitative agreement (detection of diseased fields, localization of diseased quadrants) was assessed using Cohen's kappa.

Repeatability of VFF results (scotoma area) was analysed for the whole field and individual quadrants using intraclass correlation coefficient. Differences were analysed using the Wilcoxon Signed-rank test.

Spearman's Rho was used to assess the correlation of scotoma area on VFF with that on HVF (pattern deviation and total deviation) and severity indices of glaucoma (MD; visual field index, VFI). Student's $t$ test was used to compare the time taken for HVF versus VFF.

\section{Results}

\section{Patient demographics}

A total of 96 subjects (96 eyes) were included in the study, with 66 glaucoma subjects (66 eyes) and 30 controls (30 eyes). The mean age of each group was $67.1 \pm 11.1$ and $57.6 \pm 12.4$ years, respectively. Detailed demographic data and clinical parameters were shown in Table 1.

\section{VFF versus HVF}

Data and analysis for each phase were shown in Table 2.

\section{Phase 1}

Four stimulus sizes were compared: $2 \times 2,4 \times 4,8 \times 8$, and $16 \times 16 \mathrm{px}$ (Table $2 \mathrm{a})$. VFF was able to detect VF loss in $85.0 \%, 86.2 \%, 84.2 \%$, and $78.6 \%$ of the subjects, respectively. Correspondence of diseased quadrants between HVF 

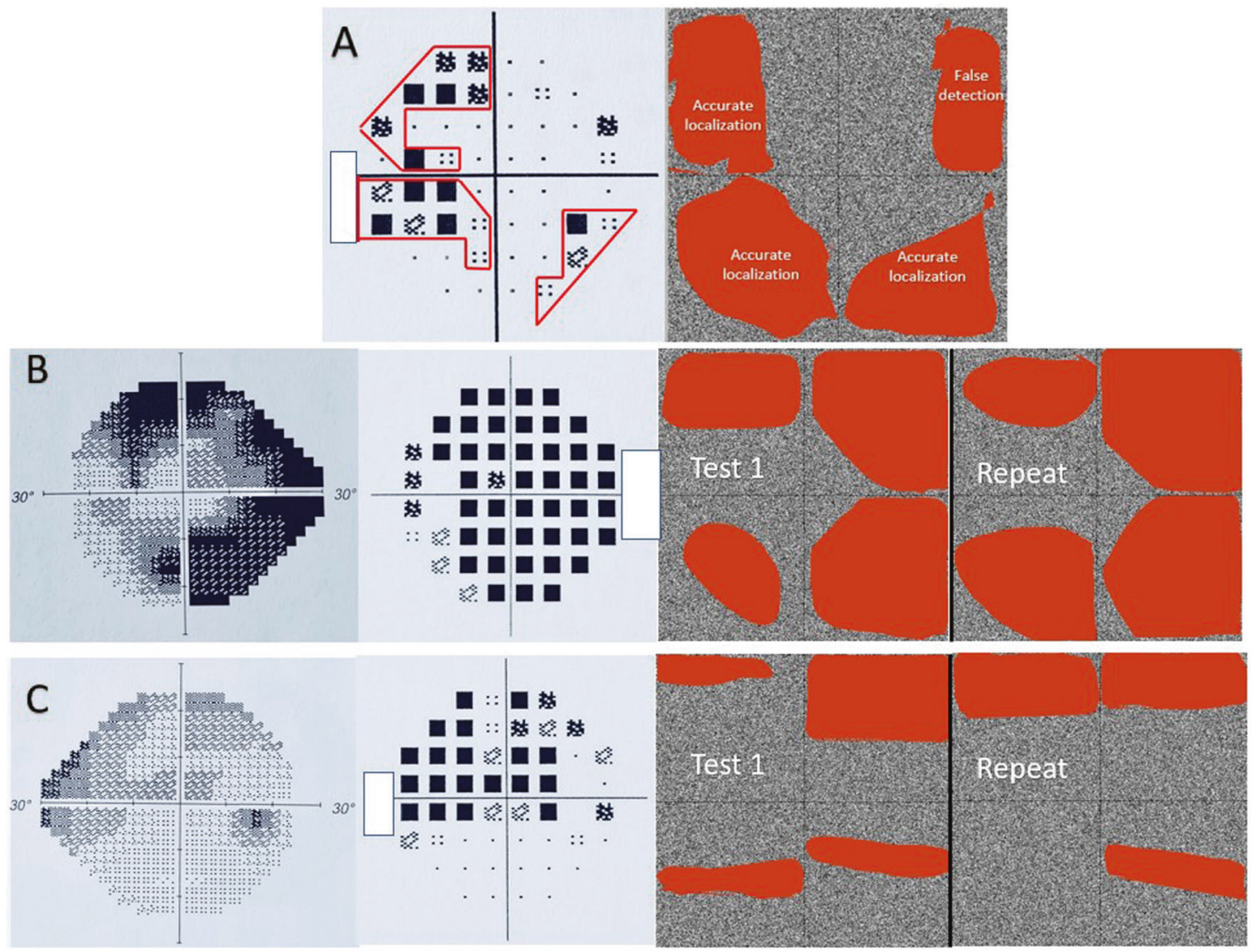

Fig. 3 Compilation of HVF pattern deviation plots, grey scale, and HVF results. A Example of false localization of scotoma occurring in the superotemporal quadrant. B Example of excellent repeatability between VFF tests, demonstrating resemblance with grey scale and pattern deviation probability plots. C Example of non-perfect correspondence between test and retest, with the repeated assessment missing a scotoma located in the inferonasal quadrant. Correspondence is still observed between VFF, grey scale and pattern deviation probability plot.

Table 1 Demographic data and test findings of study subjects $(n=96)$.

\begin{tabular}{|c|c|c|c|c|c|c|c|c|c|c|}
\hline & \multirow{2}{*}{$\begin{array}{l}\text { Type of } \\
\text { subjects }\end{array}$} & \multirow{2}{*}{$\begin{array}{l}\text { Number of } \\
\text { subjects }\end{array}$} & \multirow{2}{*}{$\begin{array}{l}\text { Mean age } \pm \\
\text { SD, years }\end{array}$} & \multirow[t]{2}{*}{$p$ value } & \multicolumn{2}{|c|}{ Gender } & \multicolumn{2}{|l|}{ Eye } & \multirow{2}{*}{$\begin{array}{l}\text { Median VFI } \\
\text { (IQR), \% }\end{array}$} & \multirow{2}{*}{$\begin{array}{l}\text { Median MD } \\
\text { (IQR), dB }\end{array}$} \\
\hline & & & & & Male & Female & Right & Left & & \\
\hline \multirow[t]{2}{*}{ Overall } & Glaucoma & 66 & $67.1 \pm 11.1$ & $<0.001$ & 42 & 24 & 40 & 26 & 78.5 (25.5) & $-8.22(0.94)$ \\
\hline & Control & 30 & $57.5 \pm 12.4$ & & 6 & 24 & 19 & 11 & $99.0(2.0)$ & $-0.45(2.08)$ \\
\hline \multirow[t]{2}{*}{ Phase 1} & Glaucoma & 29 & $67.3 \pm 11.3$ & - & 19 & 10 & 19 & 10 & $76.0(33.0)$ & $-10.0(12.0)$ \\
\hline & Control & - & - & - & - & - & - & - & - & - \\
\hline \multirow[t]{2}{*}{ Phase 2} & Glaucoma & 17 & $67.2 \pm 13.3$ & - & 7 & 10 & 10 & 7 & $82.0(24.0)$ & $-7.14(7.95)$ \\
\hline & Control & - & - & - & - & - & - & - & - & - \\
\hline \multirow[t]{2}{*}{ Phase 3} & Glaucoma & 57 & $67.7 \pm 11.0$ & $<0.001$ & 34 & 23 & 34 & 23 & $79.0(27.0)$ & $-8.20(9.20)$ \\
\hline & Control & 30 & $57.5 \pm 12.4$ & & 6 & 24 & 19 & 11 & $99.0(2.0)$ & $-0.50(1.30)$ \\
\hline
\end{tabular}

Age is presented as mean \pm standard deviation (SD), evaluated with the Student's $t$ test for independent samples. Mean deviation and visual field index are presented as median with interquartile range (IQR). Categorical variables are presented as absolute values and percentage of total. Phase 3 subjects contain those tested with best combination from the earlier 2 phases.

and VFF was $81.2 \%, 69.6 \%, 59.4 \%$, and $50.0 \%$, respectively. VFF falsely reported scotomas in $50.0 \%, 57.1 \%$, $50.0 \%$, and $60.0 \%$ of the non-diseased quadrants, respectively. The $2 \times 2-p x$ stimulus size had good detection rate and was the most accurate.

\section{Phase 2}

Three frequencies were compared: 10,20 , and $30 \mathrm{~Hz}$ (Table 2a). VFF was able to detect VF loss in $57.1 \%, 82.3 \%$, and $89.2 \%$ of the subjects, respectively. Correspondence of 
Table 2 Analysis of quantitative and qualitative data obtained from all 3 phases of study.

(a) Phases 1 (pixel size) and 2 (frequency) results of diseased field detection and diseased quadrant localization.

\begin{tabular}{|c|c|c|c|c|}
\hline & \multirow[t]{2}{*}{ Number of subjects } & \multirow[t]{2}{*}{ Diseased field detected $(\%)$} & \multicolumn{2}{|c|}{ Diseased quadrant localized (\%) } \\
\hline & & & Accurate & False \\
\hline \multicolumn{5}{|c|}{ Pixel size (px) } \\
\hline $2 \times 2$ & 20 & 85.0 & 81.2 & 45.5 \\
\hline $4 \times 4$ & 30 & 86.2 & 69.6 & 57.1 \\
\hline $8 \times 8$ & 19 & 84.2 & 59.4 & 50.0 \\
\hline $16 \times 16$ & 14 & 78.8 & 50.0 & 60.0 \\
\hline \multicolumn{5}{|c|}{ Frequency $(\mathrm{Hz})$} \\
\hline 10 & 14 & 57.1 & 31.9 & 0 \\
\hline 20 & 17 & 82.3 & 57.4 & 10.0 \\
\hline 30 & 37 & 89.2 & 77.2 & 52.4 \\
\hline
\end{tabular}

(b) Phase 3 (best combination: $2 \times 2,30 \mathrm{~Hz}$, BW stimulus) comparison of HVF and VFF test durations and scotoma areas.

\begin{tabular}{llll}
\hline & HVF & VFF & $p$ value \\
\hline $\begin{array}{l}\text { Mean test duration } \pm \text { SD, min } \\
\text { Glaucoma }\end{array}$ & $6.92 \pm 1.12$ & $3.60 \pm 1.85$ & $<0.01$ \\
$\quad$ Control & $5.16 \pm 0.727$ & $1.12 \pm 0.486$ & $<0.01$ \\
Median total scotoma area (IQR), $\%$ & & & $<0.01$ \\
Glaucoma & $44.0(30.0)^{\mathrm{a}}$ & $21.0(27.1)$ & $<0.01$ \\
Glaucoma & $85.4(36.6)^{\mathrm{b}}$ & $21.0(27.1)$ & - \\
Control & 0 & 0 & \\
\hline
\end{tabular}

(c) Phase 3 (best combination: $2 \times 2,30 \mathrm{~Hz}$, BW stimulus) Cohen's kappa calculation with $95 \%$ confidence interval (CI).

\begin{tabular}{|c|c|c|}
\hline \multirow[t]{2}{*}{ Whole field (PD and TD) } & \multicolumn{2}{|c|}{ HVF (gold standard) } \\
\hline & Abnormal $(+)$ & Normal (-) \\
\hline \multicolumn{3}{|l|}{ VFF } \\
\hline Abnormal (+) & 52 & 1 \\
\hline Normal (-) & 5 & 29 \\
\hline Kappa (95\% CI) & 0.85 & $(0.74-0.97)$ \\
\hline \multirow[t]{2}{*}{ Quadrant (PD) } & \multicolumn{2}{|c|}{ HVF (gold standard) } \\
\hline & Abnormal (+) & Normal (-) \\
\hline \multicolumn{3}{|l|}{ VFF } \\
\hline Abnormal (+) & 146 & 30 \\
\hline Normal (-) & 38 & 134 \\
\hline Kappa $(95 \%$ CI) & 0.61 & $(0.53-0.69)$ \\
\hline \multirow[t]{2}{*}{ Quadrant (TD) } & \multicolumn{2}{|c|}{ HVF (gold standard) } \\
\hline & Abnormal (+) & Normal (-) \\
\hline \multicolumn{3}{|l|}{ VFF } \\
\hline Abnormal (+) & 166 & 10 \\
\hline Normal (-) & 46 & 126 \\
\hline Kappa (95\% CI) & 0.68 & $(0.60-0.75)$ \\
\hline
\end{tabular}


(d) Phase 3 (best combination: $2 \times 2,30 \mathrm{~Hz}$, BW stimulus) quantitative repeatability assessment for whole field and individual quadrants

\begin{tabular}{|c|c|c|c|c|c|}
\hline & \multicolumn{3}{|c|}{ Median scotoma area (IQR), \% } & \multicolumn{2}{|l|}{ Statistical analysis } \\
\hline & VFF test 1 & VFF test 2 (repeat) & $p$ value & ICC $(95 \% \mathrm{CI})$ & $p$ value \\
\hline Number of patients tested & \multicolumn{2}{|c|}{19} & - & - & - \\
\hline Whole field & $25.8(19.2)$ & $27.8(23.7)$ & 0.396 & $0.96(0.90-0.98)$ & $<0.001$ \\
\hline \multicolumn{6}{|l|}{ Individual quadrants } \\
\hline Superotemporal & $26.4(30.1)$ & $25.6(36.9)$ & 0.811 & $0.92(0.81-0.97)$ & $<0.001$ \\
\hline Superonasal & $19.0(28.6)$ & $27.8(39.4)$ & 0.334 & $0.89(0.74-0.96)$ & $<0.001$ \\
\hline Inferotemporal & $23.6(35.4)$ & $30.4(33.7)$ & 0.352 & $0.91(0.78-0.96)$ & $<0.001$ \\
\hline Inferonasal & $23.6(35.5)$ & $27.8(51.9)$ & 0.460 & $0.82(0.59-0.93)$ & $<0.001$ \\
\hline
\end{tabular}

(e) Summary table of patient descriptors of scotomas from 37 subjects

\begin{tabular}{ll}
\hline Patient descriptors & Number of patients $(\%)$ \\
\hline Black, reduced flashing & $17(45.9)$ \\
Black, no flashing & $11(29.7)$ \\
Grey, reduced flashing & $1(2.7)$ \\
Grey, no flashing & $6(16.2)$ \\
Bright & $2(5.4)$ \\
\hline
\end{tabular}

(b) Time is expressed as mean \pm standard deviation (SD). Statistical difference assessed using the paired the Student's $t$ test. Scotoma area is expressed as median with interquartile range (IQR). Statistical difference is assessed using the Wilcoxon Signed-rank Test.

aThe usage of HVF pattern deviation plot for area calculation.

${ }^{\mathrm{b}}$ The usage of HVF total deviation plot for area calculation.

(c) VFF was compared against HVF (gold standard) in terms of the whole field and individual quadrants, for the correspondence of scotoma detection. Abnormal $(+)$ indicated the presence of scotoma. Normal $(-)$ indicated the absence of scotoma. With HVF as the gold standard, $+\mathrm{HVF}$ and + VFF indicate true positive; $+\mathrm{HVF}$ and - VFF indicate false negative; $-\mathrm{HVF}$ and - VFF indicate true negative; $-\mathrm{HVF}$ and + VFF indicate false positive. For whole-field analysis, same results were obtained for PD and TD.

$P D$ pattern deviation, $T D$ total deviation.

(d) Area of scotoma is represented by median with interquartile range (IQR), evaluated using the Wilcoxon Signed-rank Test. Intra-rater correlation coefficients (ICC) are used to assess test-retest repeatability, presented as ICC value with $95 \%$ confidence interval (CI). The differences between scotoma areas are tested using Wilcoxon Signed-rank Test.

diseased quadrants between HVF and VFF was $31.9 \%$, $57.4 \%$, and $77.2 \%$, respectively. VFF falsely reported scotomas in $0 \%, 10.0 \%$, and $52.4 \%$ of the non-diseased quadrants, respectively. The $30-\mathrm{Hz}$ stimulus frequency was the most superior in detection rate, albeit having more false-positive quadrants.

\section{Phase 3}

The empirically derived suitable setting $(2 \times 2 \mathrm{px}, 30 \mathrm{~Hz}, \mathrm{BW}$ stimulus) was tested on 57 glaucoma subjects and 30 controls. VFF tests were significantly faster than HVF SITA-Standard when testing glaucoma subjects $(3.60 \pm 1.12 \mathrm{~min}$ versus $6.92 \pm 1,84 \mathrm{~min}, p<0.001)$ and controls $(1.11 \pm 0.486 \mathrm{~min}$ versus $5.19 \pm 0.697 \mathrm{~min}, p<0.001$ ) (Table $2 b$ ).

For whole-field analysis with reference to HVF pattern deviation, VFF detected scotomas (diseased fields) in $91.4 \%(52 / 57)$ of glaucoma subjects (true positive) and $3.3 \%(1 / 30)$ of controls (false positive). Kappa for wholefield analysis was 0.86 (Table $2 \mathrm{c}$ ). The same results were observed when using the HVF total deviation as a reference.

For quadrant analysis with reference to $\mathrm{HVF}$ pattern deviation, $79.3 \%$ of the diseased quadrants were localized (Fig. 3A). VFF falsely reported scotoma in 1 control subject, localized to a single quadrant. Scotomas were also falsely reported in 29 non-diseased quadrants of 19 glaucoma subjects. However, their diseased quadrants were also accurately localized. Kappa for quadrant analysis was 0.61 (Table 2c). When using HVF total deviation for reference, $78.3 \%$ of the diseased quadrants were localized, with nine normal quadrants being falsely reported as abnormal. A kappa of 0.68 was obtained.

Bland-Altman plot demonstrated poor agreement between scotoma areas on HVF pattern deviation and VFF (Fig. 4A), as well as between HVF total deviation and VFF (Fig. 4B). Statistically significant positive correlations were seen in the overall area of VF loss measured by VFF and HVF pattern deviation ( $r=0.268, p=0.044$ ) (Fig. 4C), as well as for HVF total deviation $(r=0.294, p=0.027)$ 

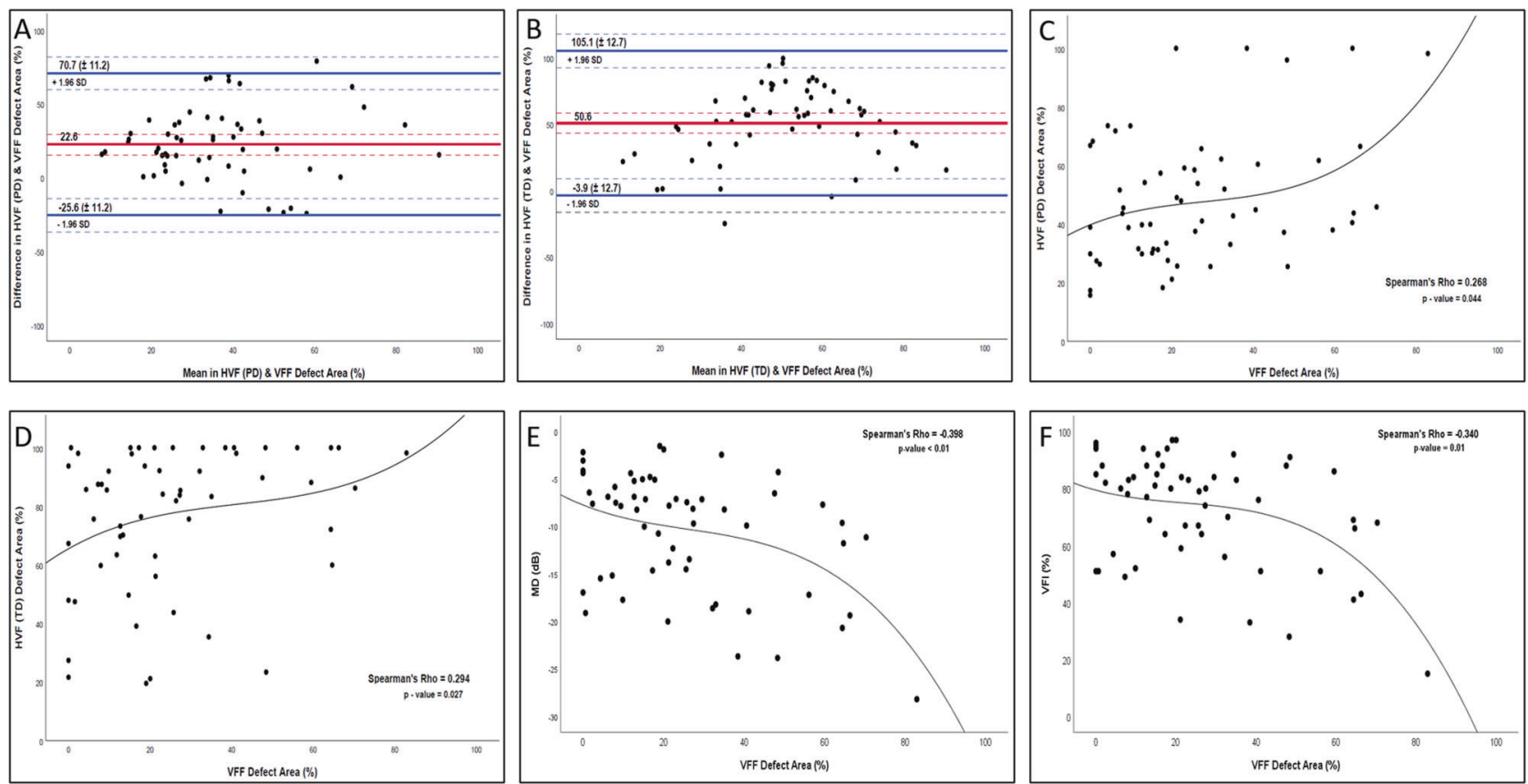

Fig. 4 Statistical analysis of scotoma area on VFF versus other parameters. A, B Bland-Altman plots of mean total area scotoma area (\%) measured by HVF and VFF versus difference (\%) between both modalities' estimates. Bias is indicated by red horizontal axis. Blue horizontal axes indicate lower and upper limits of agreement (with 95\% confidence interval), respectively. Plots were constructed

(Fig. 4D). Median scotoma area was significantly smaller on VFF than HVF pattern deviation (21.0 versus 44.0, $p<$ 0.01 ) and HVF total deviation (21.0\% versus $85.4 \%, p<$ 0.01 ). There was also a statistically significant negative correlation between the area of scotoma on VFF and VFI $(r=-0.340, p<0.01)$ and $\mathrm{MD}(r=-0.398, p<0.01)$ (Fig. 4E, F)

Repeatability was tested in 19 glaucoma subjects (Table 2d). ICC for scotoma area of the whole field $(0.96, p<$ 0.001 ) and individual quadrants (range from 0.82 to 0.92 , all $p<0.001$ ) demonstrated good to excellent test-retest reliability. No significant difference in scotoma area was found in terms of whole field and individual quadrants using the Wilcoxon Signed-rank Tests (all $p>0.05$ ). Qualitative replicability of scotoma (shape, location) was excellent (Fig. 5) only two subjects did not exhibit total correspondence (one quadrant undetected on retest) (Fig. 3B, C).

Repeatability was also tested in 28 controls. Only one reported scotoma during the test (false-positive). The scotoma was consistently located at the superior-nasal quadrant, with areas measuring 0.046 and 0.063 on repeated tests.

\section{Patient descriptors}

Response was obtained from 37 glaucoma subjects (Table 2e). Thirty-five (97.3\%) described their scotomas to for HVF pattern deviation and pattern deviation. C-F Scatter plot of total defect area on VFF (\%) versus total defect area on HVF (\%) pattern deviation and total deviation, visual field index $(\%)$ and mean deviation $(\mathrm{dB})$, respectively, with Spearman Rho calculation and trendline for monotonic relationship. PD pattern deviation, TD total deviation.

have reduced brightness and flashing. Two subjects (2.7\%) reported bright scotomas.

\section{Discussion}

This study evaluated the feasibility of VFF in detecting established cases of glaucoma with moderate or severe visual field loss. VF abnormalities were detected in $91.2 \%$ of the glaucoma subjects, with only one false-positive (3.3\%) and strong agreement with HVF [14]. Comparatively, Schiefer et al. in 1995 deduced a sensitivity of 80.7\% [15]. The difference may be attributed to Schiefer et al. recruiting subjects with various types of visual pathway lesions-noise-field campimetry was found to be poorer at detecting retro-chiasmal pathologies [8].

VFF accurately localized nearly $80.0 \%$ of the scotomas to their respective quadrants, with moderate agreement with HVF [14]. VFF's results were highly repeatable, whereby defects of similar area, shape and location could be replicated on the second attempt. Nearly all subjects perceived scotomas as areas of reduced luminance (darker) or amount of flicker, consistent with past studies [9, 16]. Patients typically described their scotomas as 'thundercloud'.

There was poor agreement between HVF and VFF in estimating the total area of defect, with VFF demonstrating 

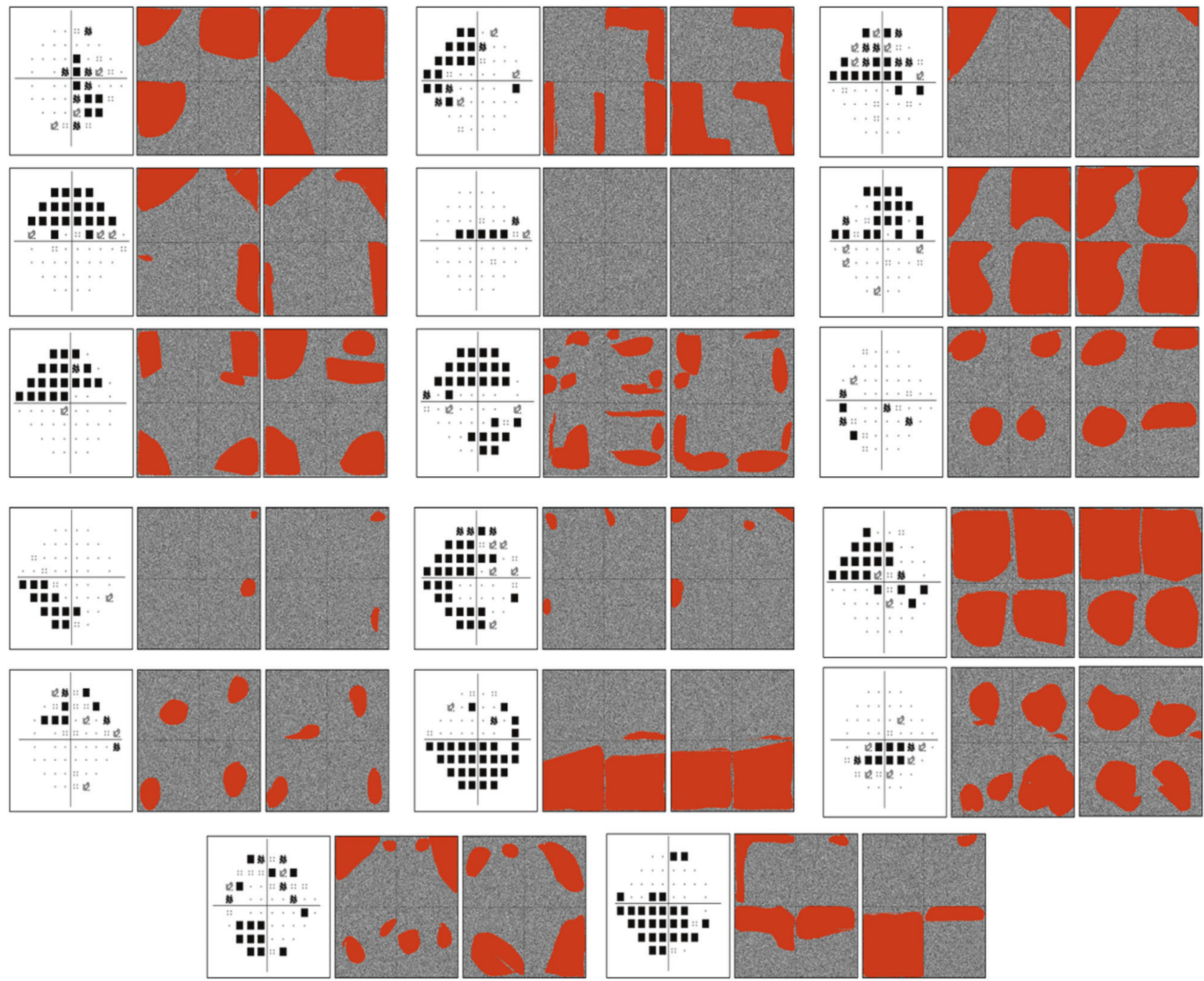

Fig. 5 Comparison of visual field defects detected by HVF and VFF. HVF and VFF results of all glaucoma subjects placed under repeatability testing, excluding the two subjects displayed in Fig. 2.

a tendency to underestimate the scotoma area. The underestimation of scotoma area using noise-field testing has been reported previously and may be attributed to fixation loss while manually outlining the scotoma, causing movement of the defect's borders [16]. Another possible reason would be our selection of surrogate markers. The 24-2 HVF only tested fifty points in the entire VF (excluding the nasalmost two points). Calculating the percentage of points with $p<5 \%$ may over-estimate the scotoma's area, as points do not truly represent areas. We included all abnormal points, including solitary ones. While these points may reflect glaucomatous damage, they do not constitute a 'scotoma'. This may suggest that VFF is better at detecting areas than discrete spots. A greater degree of underestimation of scotoma area was observed when the HVF TD plot was used as a reference standard; this can be explained by a larger defect seen on the plot, which does not account for media opacities. However, it is of note that the accuracy of VFF in detecting and localizing scotomas was unaffected by the type of HVF plot used as a reference standard. In addition, VFF exhibited statistically significant positive correlations with HVF (both pattern and total deviation) and the severity of glaucoma. This suggested that larger scotomas on VFF may indicate more severe disease.

Despite statistical significance in terms of quadrant localization and correlation with the severity of glaucoma, we must not overlook VFF's variability in test outcomes. For instance, both an HVF defect area of $50 \%$ and a MD of -10 could give a VFF result ranging from 0 to $80 \%$. There are also instances whereby scotoma localizations were completely unsuccessful. These observations may be attributable to the subjectivity of noise-field testing. Some patients reported scotomas as blurred stimulus, while others perceived a complete obliteration of flickers. It may be possible that some perceived a large defect while having a mild disease and vice versa. Fortunately, the delineated 
scotomas were repeatable across two tests, suggesting consistency in VFF test outcomes despite variability amongst patients with similar disease severity. Although current data demonstrated VFF's capability in detecting abnormalities, it also demonstrated the lack of accuracy in localizing and defining scotomas.

In terms of the recommended testing stimulus, we found better scotoma detection rates with smaller stimuli. To the best of our knowledge, there have been no similar reports on noise-field campimetry. However, with high-density stimulation (100 points), high spatial resolution perimetry (conventional) was able to detect scotomas missed with the standard HVF SITA 24-2 settings (52 points) [17]. We postulate that smaller stimuli test the same VF area more comprehensively, as the number of stimuli unit area is increased, hence improving test sensitivity. It has also been shown that bigger perimetric stimuli increase retinal sensitivity in glaucoma patients [18]. Larger stimuli may simply recruit adjacent retinal regions, allowing flicker perception through spatial summation. Hence, patients do not observe scotomas. From the psychophysics perspective, Ricco's law states that the detection threshold for a visual stimulus is dependent on its contrast/luminance and size. If the stimulus is smaller than a specific area, known as the Ricco's area, its detection will be completely dependent on luminance. Ricco's area itself enlarges physiologically with retinal eccentricity and pathologically in glaucoma [18]. Assuming equal luminance, it is therefore intuitive that smaller stimulus is less likely to be detected, causing patients to perceive scotomas instead.

VFF's performance was also enhanced at higher flicker frequencies. When testing at lower frequencies, glaucoma subjects experienced a slow flashing screen without any abnormalities. The $0 \%$ false-positive quadrants reported while using $10-\mathrm{Hz}$ stimulus may be due to its failure to elicit true scotomas. This was consistent with existing literature, which demonstrated the loss of sensitivity to high temporal frequencies $(30-40 \mathrm{~Hz})$ in glaucoma [19].

In recent years, there has been a rise of global interest in the development of iPad-based VF testing devices. Melbourne rapid fields (MRF) is an app that performs point-topoint testing of the VF (66 spots), proposed as a substitute for HVF in resourced-deprived settings [20]. MRF was validated through longitudinal studies, with its results demonstrating a strong resemblance with HVF. Notably, MRF can even generate severity indices like MD [21]. However, MRF also faced similar problems of fixation loss, false positives, and false negatives. Furthermore, the average test time is about $5.7 \mathrm{~min}$, which may be not sufficiently efficient for population screening. Currently, although MRF is a superior clinical device, our app offers a pragmatic alternative for large population screening. With simple procedures, VFF demonstrated high detection accuracy for the disease. Each assessment can be completed in 3.6 min for glaucoma subjects and $1.1 \mathrm{~min}$ for healthy controls. Our protocol may be further simplified to remove the drawing component-we can simply obtain a 'yes' or 'no' answer with regards to scotoma detection. Therefore, VFF may potentially enable more rapid disease detection. However, a well-designed study with appropriate statistical measures to compare the test times of MRF and VFF is required to arrive at a conclusion.

The main strength of our study was the simple, standardized instructions provided to all subjects; this ensured equal understanding of experimental procedures amongst participants. A deliberate effort was made to reassure all subjects that it was acceptable if no abnormalities were perceived. Assessors were masked to the HVF findings and no there was no prompting during the tests. Most subjects also had HVF performed on the day of study, ensuring that patients' visual field assessments are up-to-date.

There are limitations in our study and the VFF test itself. First, although we excluded subjects with edge effects on the visual fields used for study entry, there may still be artefactual edge effects while performing the iPad study. Second, there may be a slight displacement of the fixation target when the iPad was moved to test the different quadrants. Third, our iPad was placed on auto-brightness adjustment. Despite switching off all light sources, there may still be unpredictable inconsistencies in the background luminance of stimulus, albeit minor. Next, subjects were neither age- nor gender-matched. However, age and gender differences are unlikely to affect the results of our study. Furthermore, although VFF is faster than HVF SITAStandard, we did not compare against newer, faster settings like SITA Fast and SITA Faster. HVF defines the severity of scotomas in decibels, while VFF only indicates the presence or absence of defects. Last, most of our patients had moderate-to-severe glaucoma (MD worse than -3.0); VFF's efficacy in detecting mild diseases still awaits validation by future studies with larger sample sizes. But from a practical perspective, screening for very mild or early glaucoma using noise-field perimetry may not be realistic, as that remains a challenge even with standard automated perimetry [6].

The provision of telemedicine via iPads has been wellreceived, particularly for its convenience and effectiveness in reducing barriers to assessing specialist care [22]. Studies also reported good short-term compliance (over 6 months) with home-based visual field monitoring using tablets, and the test results were comparable to measurements in the clinic [23, 24]. Validation of VFF may provide an alternative method to facilitate the conduct of telemedicine, by offering means to remotely assess patients with VF complaints. Although VFF cannot replace HVF, its addition to normal electronic consultation offers a reasonable 
alternative that may be beneficial in the current coronavirus pandemic, as non-emergency hospital visits are discouraged. VFF may also have a potential role in community eye screening. The instructions are simple, and the test results can be interpreted easily by laypersons. Furthermore, the app is free and the user only has to bear the cost of an iPad. However, that cost may still be prohibitive, particularly in third-world countries.

We aim to further improve VFF by adding contrast testing and an automated flicker adjustment function-for example, by gradually increasing stimuli size/frequency during the same test. Testing with a wider range of stimulus may pick up more patients with visual field defects. Adding the automatic stimulus adjustment function will further improve the user experience.

In this experiment, all subjects tolerated the test well without subjective complaints. However, VFF should be used with caution in patients with epilepsy, as studies have described photo-sensitive epilepsy induced by flickers generated from light reflecting off spinning turbines [25].

Future longitudinal studies with regular testing intervals are required to elucidate VFF's true repeatability. The data may also illustrate VFF's changes (if any) with the control of glaucoma and the corresponding perimetric progression on HVF, validating its plausible role as a home-monitoring tool. VFF may potentially affect clinical decision-making. Physicians may perform gross screening on low-risk patients using VFF, before deciding on whether they should undergo HVF assessment. This may potentially reduce healthcare costs for both patients and institutions. Uveitis, neuro-ophthalmic conditions and age-related macular degeneration are also potential test targets for VFF. Finally, the true prowess of a potential screening tool is best deduced via actual application - the data obtained in a study which trials VFF in a public health screening setting would be invaluable.

In conclusion, the study of portable VF screening devices is still in its infancy. We have developed and evaluated the performance of VFF, a portable iPad-based that screens for glaucomatous visual field abnormalities using fine noisefield stimulus flickering at high frequency. Although the current model of VFF demonstrated significant positive attributes in terms of association with reference measures, test-retest repeatability and patient acceptability, there exist substantial negatives such as poor agreement with the reference measure, under/over-estimation of scotoma size and mislocalization of quadrants. These features ought to be improved in future versions. But at this early stage, VFF has fulfilled the basic requirement of detecting the presence of visual field defects in glaucoma patients while avoiding excessive false positives in normal patients. We propose VFF as a portable, accurate and pragmatic tool for glaucoma screening. Its applications in home-monitoring and clinical settings await justification through further longitudinal studies.

\section{Summary}

\section{What was known before}

- Population-based testing for visual field defects in glaucoma is limited by the lack of an efficacious, practical device.

- iPad and other personal mobile devices may be a favourable media to enable quick, accurate testing.

- There have been no previous studies evaluating the application of noise-field testing on an iPad.

\section{What this study adds}

- VFF is a quick, accurate device that detects glaucomatous visual field loss in patients using an iPad.

Acknowledgements The authors will like to acknowledge Dr Vernon KY YONG, Dr Boon Ang LIM, Dr Owen K HEE, Dr Vivien CH YIP and Dr Elton LT TAY, for their contributions to patient recruitment.

\section{Compliance with ethical standards}

Conflict of interest The authors declare no competing interests.

Publisher's note Springer Nature remains neutral with regard to jurisdictional claims in published maps and institutional affiliations.

\section{References}

1. Flaxman SR, Bourne RRA, Resnikoff S, Ackland P, Braithwaite T, Cicinelli MV, et al. Global causes of blindness and distance vision impairment 1990-2020: a systematic review and meta-analysis. Lancet Glob Health. 2017;5:e1221-e34.

2. Tham YC, Li X, Wong TY, Quigley HA, Aung T, Cheng CY. Global prevalence of glaucoma and projections of glaucoma burden through 2040: a systematic review and meta-analysis. Ophthalmology. 2014;121:2081-90.

3. Varma R, Lee PP, Goldberg I, Kotak S. An assessment of the health and economic burdens of glaucoma. Am J Ophthalmol. 2011;152:515-22.

4. Chua J, Baskaran M, Ong PG, Zheng Y, Wong TY, Aung T, et al. Prevalence, risk factors, and visual features of undiagnosed glaucoma: the Singapore Epidemiology of Eye Diseases Study. JAMA Ophthalmol. 2015;133:938-46.

5. Vijaya L, George R, Baskaran M, Arvind H, Raju P, Ramesh SV, et al. Prevalence of primary open-angle glaucoma in an urban south Indian population and comparison with a rural population. The Chennai Glaucoma Study. Ophthalmology. 2008;115:648-54.e1.

6. Mowatt G, Burr JM, Cook JA, Siddiqui MA, Ramsay C, Fraser C, et al. Screening tests for detecting open-angle glaucoma: systematic review and meta-analysis. Investigative Ophthalmol Vis Sci. 2008;49:5373-85. 
7. Wong EY, Keeffe JE, Rait JL, Vu HT, Le A, McCarty PhDC, et al. Detection of undiagnosed glaucoma by eye health professionals. Ophthalmology. 2004;111:1508-14.

8. Kolb M, Petersen D, Schiefer U, Kolb R, Skalej M. Scotoma perception in white-noise-field campimetry and postchiasmal visual pathway lesions. Ger J Ophthalmol. 1995;4:228-33.

9. Aulhorn E, Kost G. [White noise field campimetry. A new form of perimetric examination]. Klinische Monatsblatter fur Augenheilkd. 1988;192:284-8.

10. Plummer DJ, Banker A, Taskintuna I, Azen SP, Sample PA, LaBree L, et al. The utility of entoptic perimetry as a screening test for cytomegalovirus retinitis. Arch Ophthalmol. 1999;117:202-7.

11. Brown JC, Kylstra JA, Mah ML. Entoptic perimetry screening for central diabetic scotomas and macular edema. Ophthalmology. 2000;107:755-9.

12. Plummer DJ, Lopez A, Azen SP, LaBree L, Bartsch D-UG, Sadun AA, et al. Correlation between static automated and scanning laser entoptic perimetry in normal subjects and glaucoma patients. Ophthalmology. 2000;107:1693-701.

13. Landers J, Sharma A, Goldberg I, Graham S. A comparison of perimetric results with the Medmont and Humphrey perimeters. Br J Ophthalmol. 2003;87:690-4.

14. McHugh ML. Interrater reliability: the kappa statistic. Biochem Med. 2012;22:276-82.

15. Schiefer U, Pfau U, Selbmann HK, Wilhelm H, Zrenner E. [Sensitivity and specificity of masked field campimetry]. Der Ophthalmologe: Z der Dtsch Ophthalmologischen Ges. 1995;92:156-67.

16. Bachmann G, Fahle M. Component perimetry: a fast method to detect visual field defects caused by brain lesions. Investigative Ophthalmol Vis Sci. 2000;41:2870-86.

17. Westcott MC, Garway-Heath DF, Fitzke FW, Kamal D, Hitchings RA. Use of high spatial resolution perimetry to identify scotomata not apparent with conventional perimetry in the nasal field of glaucomatous subjects. Br J Ophthalmol. 2002;86:761-6.

18. Redmond T, Garway-Heath DF, Zlatkova MB, Anderson RS. Sensitivity loss in early glaucoma can be mapped to an enlargement of the area of complete spatial summation. Investig Ophthalmol Vis Sci. 2010;51:6540-8.

19. Tyler CW. Specific deficits of flicker sensitivity in glaucoma and ocular hypertension. Investig Ophthalmol Vis Sci. 1981;20: 204-12.

20. Vingrys AJ, Healey JK, Liew S, Saharinen V, Tran M, Wu W, et al. Validation of a tablet as a tangent perimeter. Transl Vis Sci Technol. 2016;5:3.

21. Kong YXG, He M, Crowston JG, Vingrys AJ. A comparison of perimetric results from a tablet perimeter and Humphrey Field Analyzer in glaucoma patients. Transl Vis Sci Technol. 2016;5:2.

22. Sechrist S, Lavoie S, Khong C-M, Dirlikov B, Shem K. Telemedicine using an iPad in the spinal cord injury population: a utility and patient satisfaction study. Spinal Cord Ser Cases. 2018;4:71.

23. Jones PR, Campbell P, Callaghan T, Jones L, Asfaw DS, Edgar DF, et al. Glaucoma home monitoring using a tablet-based visual field test (Eyecatcher): an assessment of accuracy and adherence over 6 months. Am J Ophthalmol. 2021;223:42-52.

24. Prea SM, Kong GYX, Guymer RH, Vingrys AJ. Uptake, persistence, and performance of weekly home monitoring of visual field in a large cohort of patients with glaucoma. Am J Ophthalmol. 2021;223:286-95.

25. Harding G, Harding P, Wilkins A. Wind turbines, flicker, and photosensitive epilepsy: characterizing the flashing that may precipitate seizures and optimizing guidelines to prevent them. Epilepsia. 2008;49:1095-8. 